
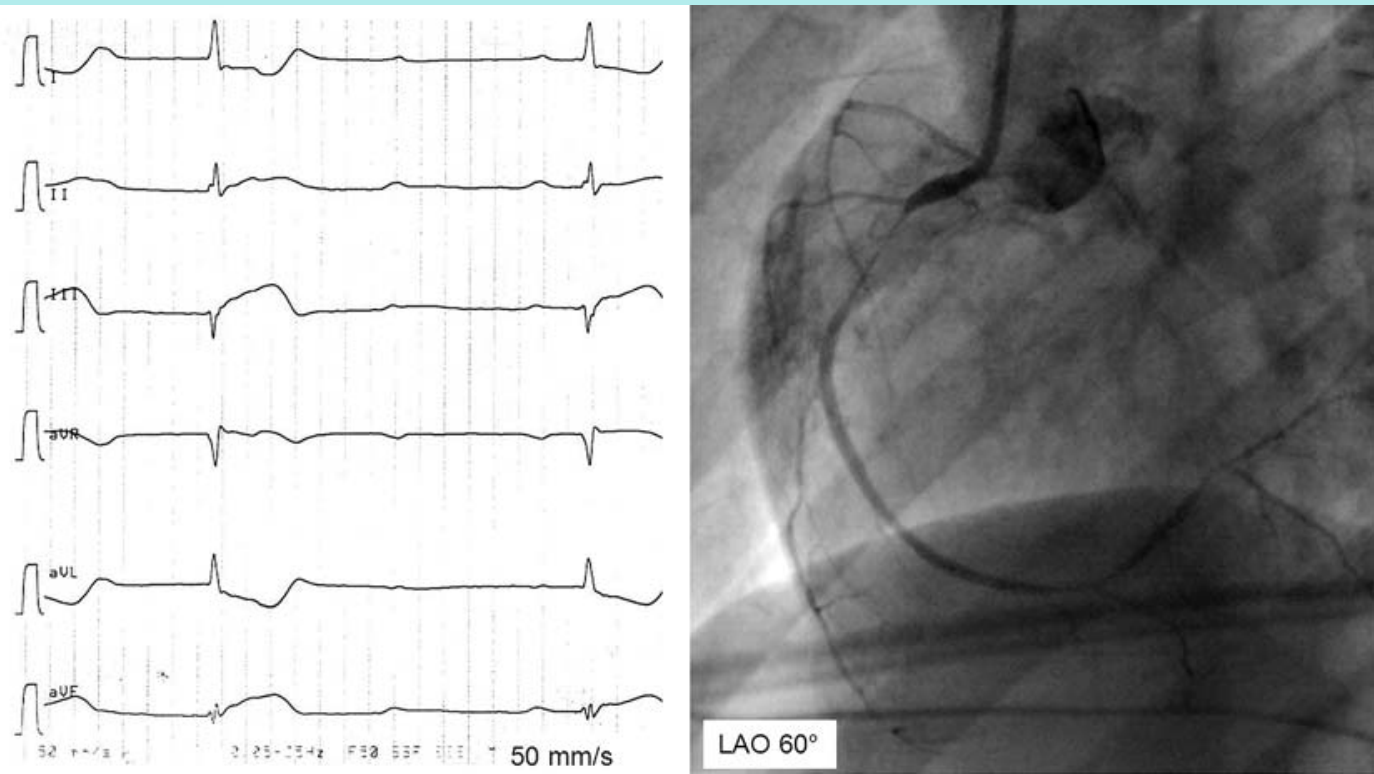

Abb.1 links Elektrokardiogramm bei Aufnahme. rechts Koronarangiographie der rechten Koronararterie.

\section{G. Michels}

B. Krausgrill

M. von Bergwelt-Baildon

\section{R. Pfister}

${ }^{1}$ Klinik III für Innere Medizin,

Universität zu Köln, Köln

2Klinik I für Innere Medizin,

Universität zu Köln, Köln

Bibliografie

DOI 10.1055/s-0032-1327307

Dtsch Med Wochenschr 2013;

138: 129-130 - (c) Georg Thie-

me Verlag KG · Stuttgart · New

York · ISSN 0012-0472

Korrespondenz

Priv.-Doz. Dr. Guido Michels

Klinik III für Innere Medizin der

Universität zu Köln

Kerpener Str. 62

50937 Köln

Tel. 0221/478-32401

Fax 0221/478-32400

eMail guido.michels@

uk-koeln.de
Die 24-jährige Patientin stellte sich wegen Angina-pectoris-Beschwerden und Synkopen in unserer Klinik vor. Anamnestisch bestanden seit Monaten intermittierende pektanginöse Beschwerden. Das EKG bei Aufnahme zeigte vorliegende Befunde. In der Laborchemie fielen eine Erhöhung der Herzenzyme (CK 259 U/l, CK-MB 38 U/l) und des Troponin T $(0,2 \mu \mathrm{g} / \mathrm{l})$ auf. Die umgehende Koronarangiographie ergab einen unauffälligen Befund der linken Koronararterie. Die rechte Koronararterie zeigte folgenden pathologischen Befund.

\section{Das Aufnahme-EKG zeigte zwei und die Koronarangiographie einen pathologischen Befund.}

\section{Welche sind das?}

Erlaubt dieser Befund eine Diagnose?

Wenn ja, welche?

Sind Differenzialdiagnosen möglich?

Wenn ja, welche? 

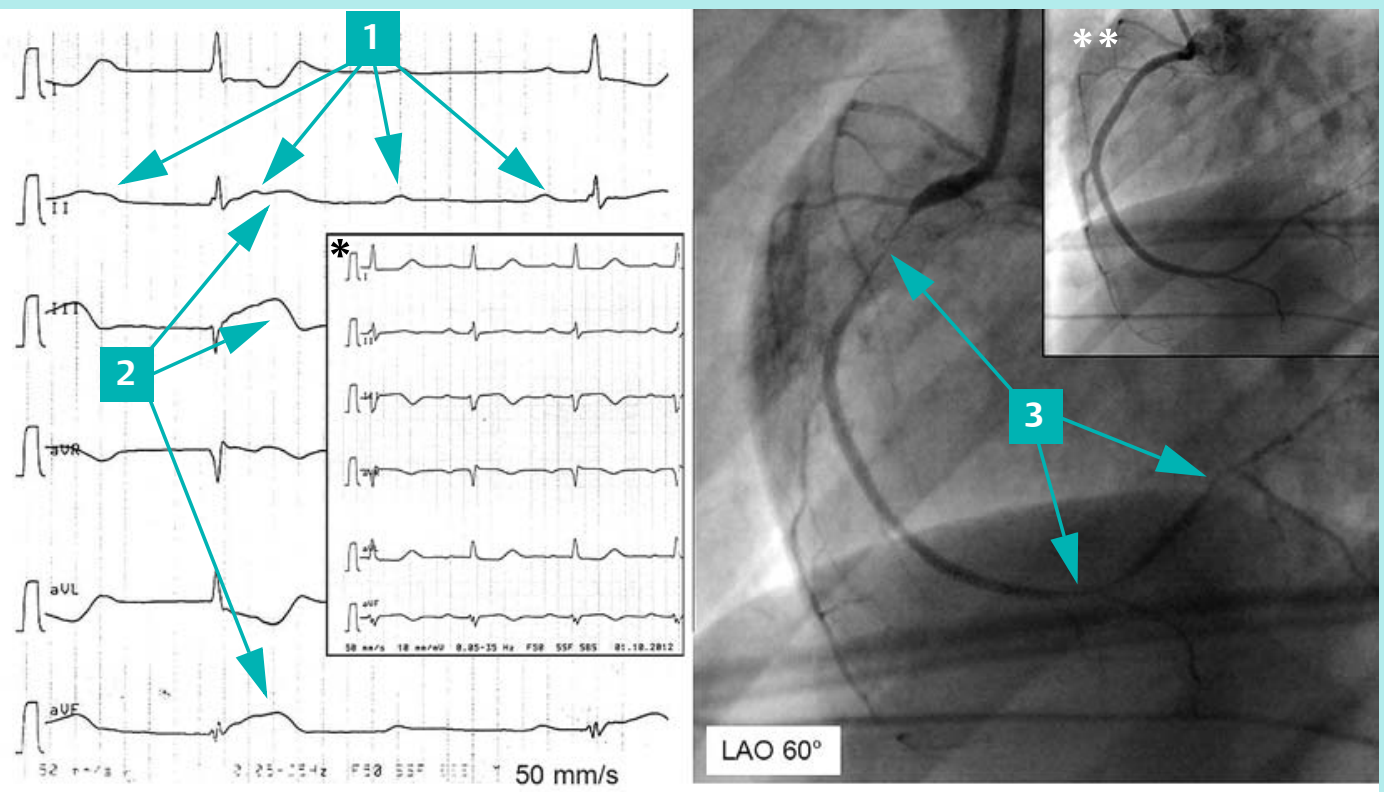

Abb.2 a Elektrokardiogramm bei Aufnahme ( ${ }^{*}$ nach intrakoronarer Nitroglyceringabe). b Koronarangiographie der rechten Koronararterie ( ${ }^{* *}$ nach intrakoronarer Nitroglyceringabe).

\section{- Befunde}

1. AV-Blockierung Grad III (52/min; durchlaufende P-Wellen)

2. Monophasische ST-Streckenhebung in II, III und aVF

3. Filiforme Stenosierungen der rechten Koronararterie

\section{Diagnose}

- Koronarspasmus der rechten Koronararterie

\section{Differenzialdiagnosen}

- Akutes Koronarsyndrom durch eine atherosklerotische Koronarstenose, Thromben oder Embolien

- Nicht-ischämischer Koronarspasmus im Rahmen einer Herzkatheteruntersuchung durch mechanische Irritation des Gefäßendothels durch z.B. Koronardrähte

\section{Erläuterung}

Das akute Koronarsyndrom ohne das Vorliegen einer obstruktiven koronaren Herzerkrankung tritt in 5 bis $10 \%$ aller Fälle - insbesondere bei Frauen - auf [1, 2,3]. Im Jahre 1959 berichteten Prinzmetal und Mitarbeiter [3] über eine „variant form of angina“, eine sogenannte Variant-Angina, als Ursache für pektanginöse Beschwerden. Leitsymptom ist die Ruhe-Angina in frühen Morgenstunden. Als Koronarspasmus wird seither eine funktionelle und reversible Stenosierung der Koronararterien verstanden. Als Ursache des Koronarspasmus werden eine chronische Endothelschädigung mit endothelialer Dysfunktion und ein Mangel an Stickstoffmonoxid mit Hyperreaktivität von glatten Gefäßmuskelzellen hauptverantwortlich gemacht [2].

Unser Fall beschreibt einen extremen spontanen Koronarspasmus der rechten Koronararterie mit der Folge eines ST-Hebungsinfarkts und totalem AV-Block. Nach intrakoronarer Nitroglyceringabe verschwanden sowohl die Beschwerden als auch die EKGVeränderungen und die filiformen Stenosierungen der rechten Koronararterie (O Abb. 2a* und 2b**). Zum direkten Nachweis von Koronarspasmen kann der intrakoronare Provokationstest mit Acetylcholin herangezogen werden. Die anschließende intrakoronare Applikation von Nitraten führt wiederum zur Aufhebung der vasospastischen Reaktion. Nach Diagnosesicherung wurde bei unserer Patientin eine Therapie mit einem Kalzium-Antagonisten vom 1,4Dihydropyridin-Typ (Amlodipin) eingeleitet.

Literatur

1 JCS Joint Working Group. Guidelines for diagnosis and treatment of patients with vasospastic angina (coronary spastic angina) (JCS 2008). Circ J 2010; 74: 1745-1762

2 Lanza GA, Careri G, Crea F. Mechanisms of coronary artery spasm. Circulation 2011; 124 : 1774-1782

3 Prinzmetal $M$ et al. A variant form of angina pectoris. Am J Med 1959; 27: 375-388 